\title{
BMJ Open Development and evaluation of a patient decision aid for patients considering ongoing medical or surgical treatment options for ulcerative colitis using a mixed-methods approach: protocol for DISCUSS study
}

\author{
Daniel Mark Baker (D) , ${ }^{1}$ Matthew James Lee (D) , ${ }^{2}$ Anne-Mairead Folan, ${ }^{3}$ \\ Sue Blackwell, ${ }^{4}$ Kerry Robinson, ${ }^{5}$ Rebecca Wootton, ${ }^{6}$ Shaji Sebastian, ${ }^{7}$ \\ Steven R Brown, ${ }^{2}$ Georgina Louise Jones, ${ }^{8}$ Alan J Lobo ${ }^{9}$
}

To cite: Baker DM, Lee MJ, Folan A-M, et al. Development and evaluation of a patient decision aid for patients considering ongoing medical or surgical treatment options for ulcerative colitis using a mixedmethods approach: protocol for DISCUSS study. BMJ Open 2020;10:e031845. doi:10.1136/ bmjopen-2019-031845

- Prepublication history and additional material for this paper are available online. To view these files, please visit the journal online (http://dx.doi org/10.1136/bmjopen-2019031845).

Received 21 May 2019 Revised 13 0ctober 2019 Accepted 20 November 2019

Check for updates

(c) Author(s) (or their employer(s)) 2020. Re-use permitted under CC BY-NC. No commercial re-use. See rights and permissions. Published by BMJ.

For numbered affiliations see end of article.

Correspondence to

Professor Alan J Lobo

alan.lobo@nhs.net

\section{ABSTRACT}

Introduction Approximately $20 \%-30 \%$ of patients with ulcerative colitis (UC) require surgery, the majority of these being elective due to chronic symptoms refractory to medical treatment. The decision for surgery is difficult and dependent on patient preferences. Current resources for patients considering surgery have been found not to meet minimum international standards. The overall aim of the 'DISCUSS' study is to develop and evaluate a new patient decision aid (PtDA) for patients considering surgery for UC created in line with international minimum standards. Methods and analysis This is a prospective mixedmethods study of adults (18+ years) who are considering surgical intervention for UC across two regional centres in Yorkshire, UK. This study is in three stages. In stage 1 we will develop the PtDA and its content via systematic reviews and a patient questionnaire. In stage 2 we will assess the face validity of the PtDA using mixed-methods on key stakeholders using both semistructured interviews and questionnaires, following which the PtDA will be refined. In stage 3 we will assess the acceptability of using the PtDA in clinical practice. This will use a mixed-methods approach on clinicians and patients who are considering undergoing elective surgery. Questionnaires including the Preparation for Decision-Making Scale, a measure of anxiety and decisional conflict will be analysed at two timepoints using paired sample t-tests and Cls. Interviews with patients and clinicians will be analysed using thematic analysis.

Ethics and dissemination Research ethics approval from North East-Tyne \& Wear South Research Ethics Committee (Ref: 19/NE/0073) and Health Research Authority approval (Ref: 257044) have been granted. Results will be published in open access peer-reviewed journals, presented in conferences and distributed through the Crohn's and Colitis UK charity. External endorsement will be sought from the International Patient Decision Aid Standards Collaboration inventory of PtDAs.

PROSPERO registration number CRD42018115513, CRD42019126186, CRD42019125193.
Strengths and limitations of this study

- This study will develop and evaluate a new patient decision aid (PtDA) for patients considering surgery for ulcerative colitis which will meet minimum international standards. This is a preliminary pilot study.

- This study will provide evidence for its acceptability and value to patients in routine clinical practice when considering surgery for ulcerative colitis.

- This study will also provide evidence of the acceptability of the PtDA from the clinicians' perspective and the feasibility of use in routine clinical practice.

- This study will not provide evidence on the value of the PtDA nationally. However, it will provide evidence across two large regional centres in Yorkshire which may be used to form the study design of a national evaluation.

\section{INTRODUCTION}

The rationale for the study

Ulcerative colitis (UC) is a chronic, relapsing and remitting inflammatory condition of the colon and rectum. It causes debilitating symptoms such as bleeding per rectum, increased stool frequency, abdominal pain and tenesmus. ${ }^{1}$ Symptoms can be managed using medical therapies such as aminosalicylates, corticosteroids, biologic agents (anti-tumour necrosis factor- $\alpha$ or anti-integrin) and tofacitinib. ${ }^{2-5}$ However, approximately $20 \%-30 \%$ of patients with UC will require surgery during their disease course. ${ }^{6}$ A minority of patients will require emergency surgery-but the majority of patients have elective surgery due to chronic symptoms, refractory to medical treatment. ${ }^{7}$ Individuals therefore make a choice, or series of choices, to continue with 
medical treatment or undergo surgery. Surgery may be undertaken with the intention of proceeding to further reconstructive surgery to restore continuity of the gastrointestinal tract, or remaining with a permanent ileostomy.

The decision to opt for elective surgery is described as preference sensitive as the preferred treatment option is dependent on patient preferences due to clinical equipoise between the options. ${ }^{8}$ The same can be said for the decision between reconstructive surgery versus permanent ileostomy. The impact on lifestyle of these two choices cannot be understated-reconstructive surgery will avoid a stoma, with an acceptance of potential complications such as increased stool frequency, pouchitis or faecal incontinence. ${ }^{9}$ A stoma may offer more control over excretory functions, but is associated with complications such as parastomal hernia, as well as psychological sequelae. ${ }^{10}{ }^{11}$ When selecting a treatment option, it is clear that the patient must select the option based on their preferences.

Shared decision-making is a process whereby clinicians share information about treatment options, empowering the patient to make a decision based on their preferences. ${ }^{12}$ Providing clear and balanced preoperative information is a major prerequisite to informed decisionmaking. ${ }^{12} 13$ The National Institute for Health and Care Excellence guidelines for UC emphasise the importance of providing such information, but note that no highquality studies assessing the desired content of information were available on which to base recommendations. ${ }^{14}$ As such clinicians lack guidance on patient informational preferences on which to base discussions during consultations. Clinician preferences can be misaligned with patient preferences, especially when considering surgical options for UC, ${ }^{15}$ forming a barrier to decision-making in this population. Preoperative discussions may also be limited by 'implicit persuasion'-a process whereby clinicians subconsciously place greater emphasis on treatment options they believe are suited to the patient. ${ }^{16}$ Lack of time in clinic, as well as the lack of guidance on the content of preoperative consultations, may provide limitations in the shared decision-making process for patients with UC. ${ }^{17}$

\section{Patient decision aids}

Patient decision aids (PtDA) are specially created tools which aim to improve patient knowledge and aid decision-making. ${ }^{19} 19$ They are evidence based, using the most up-to-date clinical evidence, studies assessing patient informational preferences and evidence on how patients make decisions. ${ }^{20}$ PtDAs can be used within the clinical encounter, by the clinician, to provide structure to consultations, but also by the patients outside the encounter to aid their deliberation-a key step to informed consent. ${ }^{13}$

A systematic review of PtDAs in surgery has illustrated their role in improving knowledge, reducing decisional conflict and increasing patient input into decisionmaking. ${ }^{21}$ A satisfactory PtDA for patients considering surgery for UC is not currently available. The single aid registered on the Decision Aids Library Inventory ${ }^{22}$ does not meet minimum standards laid out by the International Patient Decision Aid Standards (IPDAS), ${ }^{23}$ which have established clear guidance for the systematic development of a PtDA. ${ }^{24}$ This can be summarised into three stages, as previously described by members of our research group, ${ }^{25}$ and forms the methodology behind this protocol. It is therefore proposed that a new PtDA for patients considering surgery for UC, created in line with minimum standards, will work towards filling an informational need for both patients and clinicians. ${ }^{1426}$

\section{Aims and objectives}

The aim of this research is to develop, assess and validate a PtDA for patients considering elective surgery for UC; that is, whether to continue with their current medical treatment, or whether to undergo surgery. This will also include information on the different surgical options (mainly permanent stomas vs reconstructive surgery) as this may influence the overall decision to undergo surgery. We will do this in line with the systematic development process specified by IPDAS, ensuring the decision aid meets minimum standards. ${ }^{24} 27$

The study objectives are to:

1. Develop a decision aid for use by gastroenterology and colorectal surgery teams (consultant surgeons/gastroenterologists, stoma/inflammatory bowel disease (IBD) nurses) to support adult patients ( $>18$ years of age) in their decision about elective surgery, and the surgical option they may wish to opt for.

2. Assess the face validity of the decision aid to support patients make an informed decision about their preferred treatment option.

3. Pilot an evaluation study for the acceptability of the decision aid in clinical practice. This will use a mixedmethods approach to capture the views of:

A. Patients making the treatment decision.

B. Gastroenterology and colorectal surgery health professionals supporting the patient through their decision.

It is anticipated the evaluation of the PtDA will determine whether the administration of a PtDA in the treatment pathway will better support patients in their treatment decisions, as well as providing structure and guidance to consultations with patients.

\section{METHODS AND ANALYSIS}

The methodology used for this research has been adapted from a previously published protocol to develop a PtDA for women with cancer to help them make fertility preservation treatment decisions (Cancer, Fertility and Me), led by a member of our research team (GLJ) and funded by Yorkshire Cancer Research (S391). ${ }^{25}$ The protocol also follows established guidance from IPDAS and other recommended guidance regarding the development of PtDA. ${ }^{24} 27$ The study process is summarised in figure 1. 

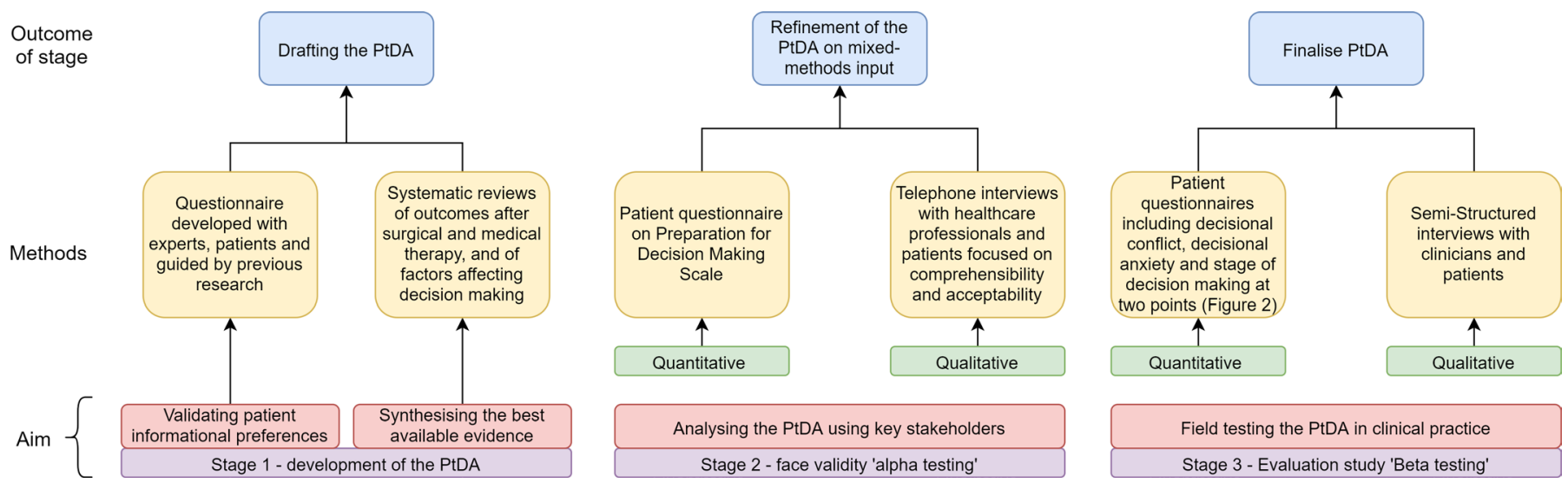

Figure 1 Summary of the study stages. PtDA, patient decision aid.

A steering group with relevant expertise to support PtDA development is essential. ${ }^{24}$ Therefore, a steering group was created prior to protocol development and submission for grant funding to ensure all stakeholders were represented. The steering group consists of: specialist surgical and gastroenterology clinicians, health psychologists with expertise in decision-making, IBD/ stoma nurses, a medical student and patient representatives. All sections of the protocol have been reviewed and discussed by the steering group. The PtDA will be developed across two regional centres (Sheffield Teaching Hospitals and Hull and East Yorkshire Hospitals). The steering group will hold regular meetings during stage 1 to decide the content and design of the aid, and will hold regular meetings at important stages thereafter. Ethics approval was granted on 13 March 2019.

\section{Design}

Stage 1: development of the PtDA

Validating patient informational preferences

Our group has already carried out qualitative work exploring patient informational preferences when considering surgery. ${ }^{26}$ These results will be validated on a national scale by a questionnaire using established methodology. ${ }^{15} 28$ Questionnaire content will include demographic data, the control preferences scale ${ }^{29}$ and questions about the preferred content and format of preoperative information. This will provide a description of whether particular demographic groups of patients are amenable to a PtDA, and the preferred content and format of such an aid. Questionnaires will be disseminated to a number of sites through an established network of IBD researchers. Prior to questionnaire development, patients will be involved in questionnaire design and refinement via a focus group held at Sheffield Teaching Hospitals. This will help make the findings generalisable and ensure key concepts are included within the questionnaire.

\section{Synthesising the best available evidence}

This will consist of the following stages:

A. Systematic reviews of evidence about the risks and benefits of elective surgery and continued medical management will be undertaken, as well as a system- atic review on factors influencing treatment choices. A full list of inclusion and exclusion criteria for each review is listed in online supplementary appendix tables S1-S3):

- A systematic review of outcomes after surgery to inform the PtDA using the best available evidence. This has been registered on the PROSPERO (www. crd.york.ac.uk/PROSPERO) database. Methodology will include the procedures of subtotal colectomy with permanent end ileostomy, proctocolectomy, ileal-anal pouch anastomosis and ileorectal anastomosis. Ileorectal anastomosis will be included in the PtDA as our research group notes it is a procedure that is offered in some UK centres, as well as in centres outside the UK. Its inclusion is with the caveat that some centres may not offer this operation, and this will be noted in the aid. The primary outcome will be quality of life, with secondary outcomes covering a wide range of early and late complications after surgery.

- Systematic review of risks and benefits of continued medical treatment. This will inform the decision aid so that the consequences of continued medical treatment-positive and adverse-can be quantified for patients facing this choice. This has been registered on PROSPERO.

- Systematic review of the factors that may facilitate or hinder patients with UC to make medical and surgical treatment choices (eg, possible fear of a stoma) to ensure these elements are captured and addressed in the new resource. This has been registered on PROSPERO.

B. Focus groups with expert clinicians, nurses and patients regarding the optimum time to introduce the PtDA into the treatment pathway, as well as the optimum content for each group. This will be via a patient and public involvement day at Sheffield Teaching Hospitals.

\section{Drafting the PtDA}

Once the evidence has been synthesised, the steering group will meet to decide the content of the aid. The 
PtDA will be created using IPDAS guidance, ${ }^{24} 27$ including guidance on balancing options, ${ }^{30}$ risk presentation, ${ }^{30-33}$ eliciting patient values, ${ }^{34}$ use of patient stories $^{18}$ and enabling readability, ${ }^{35}{ }^{36}$ something found to be poor in the UC patient literature. ${ }^{23}$

The content of the aid will be guided by the informational preference studies and systematic reviews of evidence we complete. Significant risks, benefits and outcomes and their associated probabilities from our systematic reviews will be included. Common topics of informational preferences will be discussed by the group, and a consensus established on the inclusion. The composition of the group, with both expert clinicians and patients, will help develop content that meets the requirements of both patients and healthcare professionals.

\section{Stage 2: face validity study}

The aim of this stage is to assess the PtDA for comprehension, feasibility and acceptability using key stakeholderssometimes referred as learner verification or alpha testing. ${ }^{24} 37$ This will be done with both clinicians and patients, using qualitative methodology, and according to an established protocol, with which we have extensive experience. This will be undertaken across two large sites (Sheffield and Hull).

\section{Sample}

A purposive sample of healthcare professionals and patients not involved in the steering group will be invited to take part in the study. Purposive sampling has been chosen to ensure recruitment of a representative sample of both healthcare professionals and patients. The Healthcare Professional (HCP) sample will include colorectal surgeons, gastroenterologists, IBD nurses and stoma nurses. The patient sample will include those who opted for surgery, those who considered but declined surgery and those currently deliberating treatment options. We expect a sample size of 20 participants, with a minimum of 10 healthcare professionals and 10 patients, will be enough based on previous studies and experience. ${ }^{25}$ However, sample size will be guided by data saturation, which is in line with good qualitative methods. ${ }^{38-40}$

\section{Recruitment}

Patients will be identified through the services at the two clinical centres by clinicians and nurses. We will also advertise stage 2 of the study through the Crohn's and Colitis UK forums. Following this the contact details for consenting patients will be passed on to a trained researcher and those willing to participate will be sent the PtDA for review. Healthcare professionals will be recruited from the study sites through purposive sampling. All contact details will be stored securely at either Sheffield Teaching Hospitals or Leeds Beckett University.

\section{Data collection}

\section{Qualitative}

Consenting clinicians and patients will be posted the PtDA and given 1-2 weeks to assess the aid, with a telephone interview taking place at the end of the time period. Patients and clinicians will provide verbal feedback on the aid, focusing on its comprehensibility and ease of use. An interview schedule will be created a priori by expert members of the steering group. Interviews will be audio recorded, digitalised and transcribed for analysis.

\section{Quantitative}

Patients will also be asked to complete a Preparation for Decision-Making Questionnaire. The Preparation for Decision-Making Questionnaire is a 10-item measure which will provide a score on a scale of $0-100 .{ }^{41}$ The higher the score, the higher the perceived levels of preparation for decision-making - which will provide a validated quantitative measure of how individuals view the usefulness of the PtDA. ${ }^{41}$

\section{Data analysis}

Interviews will be transcribed and coded using NVivo V.11 computer-assisted qualitative data analysis software (QSR International, Australia). Analysis will use an inductive thematic approach, outlined by Braun and Clarke using a systematic five-step approach: familiarisation, generating initial codes, searching for themes, reviewing themes and defining and naming themes. ${ }^{40}$ The themes actively generated by the researchers from the data will be discussed by the steering group.

The steering group will subsequently refine the aid based on the results of this stage. If there are significant changes required, a second face validity study will be undertaken before progression to stage 3 .

\section{Stage 3: evaluation study}

The aim of this pilot is to field test the PtDA in clinical practice, as there may be clinical contextual factors impacting the PtDA. This is typically referred to as beta testing. ${ }^{24}$ This will follow a mixed-methods approach across the same two sites as in stage 2 . We aim to recruit 15 patients at each site for a minimum sample size of 30comparable with other PtDA pilots in the literature. ${ }^{42} \mathrm{~A}$ summary of stage 3 is provided in figure 2 .

\section{Quantitative}

\section{Sample and recruitment}

All patients over the age of 18 who receive consultation about the possibility of undergoing elective surgery will be included. This includes consultations with clinicians and specialist nurses. We will use the referral model for implementing PtDA-a process where the clinician mentions the PtDA to eligible patients during consultation about treatment options, and indicates the clinical researchers will discuss the study following the consultation should the patient consent to involvement. ${ }^{43}$ 


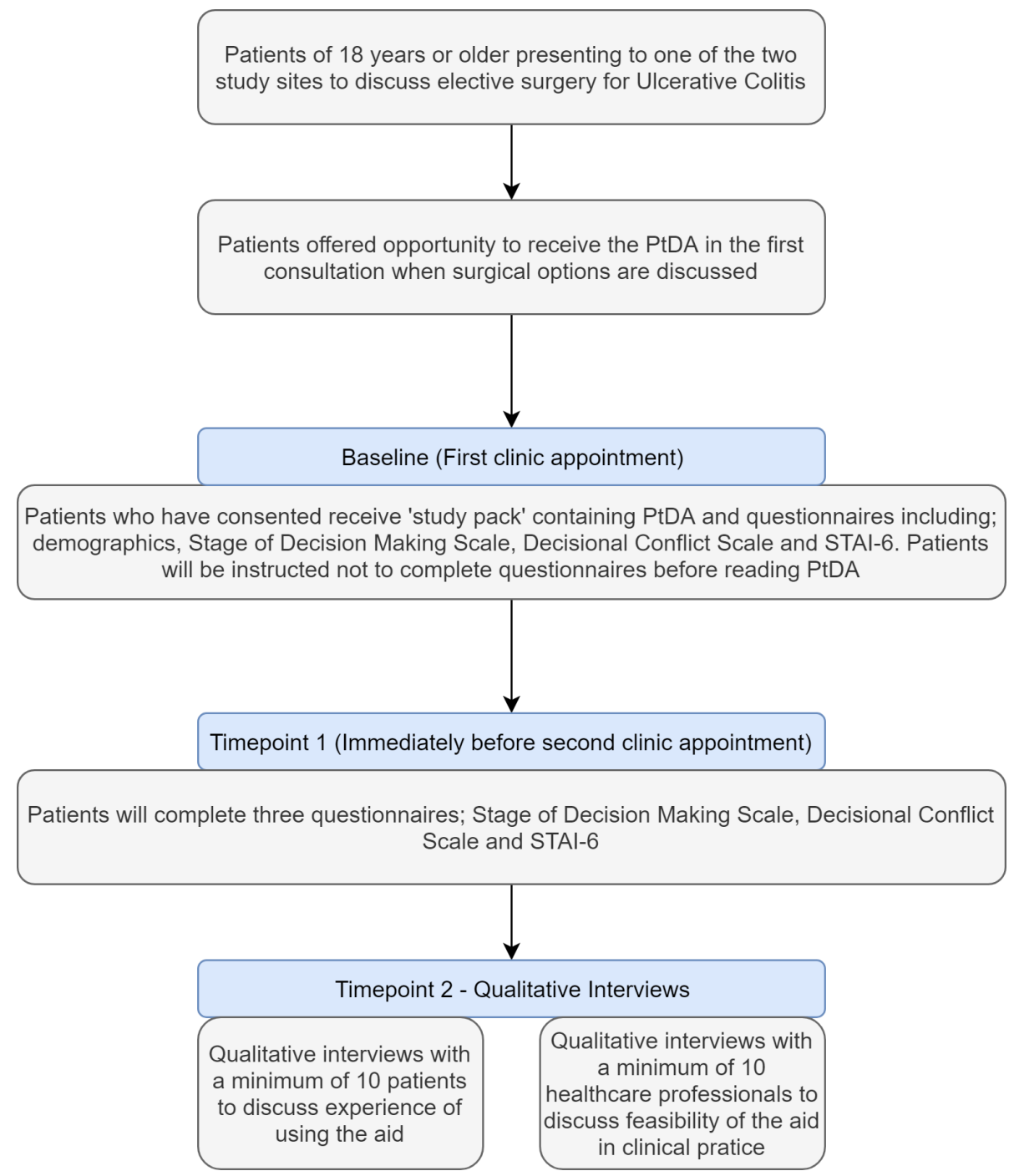

Figure 2 Summary of stage 3-evaluation study. PtDA, patient decision aid; STAI-6, Spielberger State-Trait Anxiety Inventory-6.

Eligible patients identified by the clinical team will be invited to participate by the researcher(s) with the clinical team immediately following the consultation if they are present, or within a week if the patient consents to contact outside the clinical setting by a member of the research team.

\section{Data measures and collection}

Patients will be asked to complete a series of questionnaires at baseline (first clinic appointment) before administration of the PtDA. Patients will be instructed not to open or view the PtDA before completion of the questionnaires at baseline. Patients will then complete the same questionnaires at timepoint 1 (immediately before the second clinic appointment). Questionnaires will include a measure of anxiety (Spielberger State-Trait Anxiety Inventory-6), ${ }^{44}$ Stage of Decision-Making ${ }^{45}$ and Decisional Conflict Scale ${ }^{46}$ as recommended by IPDAS. ${ }^{24}$ All questionnaires will provide a quantitative measure to allow comparison before and after use of the PtDA.
Analysis

Summary statistics will be reported for demographics and other relevant indicators. For decisional outcome measures we will use paired sample t-tests to calculate mean changes from baseline to timepoint 1 . CIs will be set at $95 \%$ a priori, meaning values will be significant if $\mathrm{p} \leq 0.05$.

\section{Qualitative}

\section{Sample and recruitment}

Healthcare professionals and patients will be asked to take part in semistructured interviews. We will undertake 10 interviews with patients and 10 with healthcare professionals (IBD/stoma nurses, gastroenterology and surgical clinicians), although this will be guided by data saturation, in line with established protocols in qualitative research. ${ }^{3840}$ The interviews will give a deeper insight into the experience of using the aid from a patient and clinician perspective in clinical practice. Clinician interviews 
will also explore views regarding the timing of the aid in the treatment pathway, establishing if the time previously eliciting in stage 1 is also the optimum time for additional sites. Patients will be recruited using purposive sampling from the sample of 30 that have taken part in the quantitative analysis of stage 3 . Prior to interview, patients will receive an information sheet, and on the day of interview the patient will be issued a consent form which will be cosigned with the interviewer.

\section{Data collection}

An interview transcript will be created by expert members of the steering group a priori. Questions will be adapted from the transcript used in stage 2 , with additional questions to add depth and clarity into the interpretation of the quantitative results. Questions will also focus on the PtDA usefulness in helping the patient decide between treatment options.

Analysis

All interviews will be transcribed and coded as per the same methods in stage 2. Framework analysis, an analysis designed specifically for applied health and policy research, ${ }^{47}$ will be used to identify recurrent themes. Recurrent themes will be discussed with the steering group and subsequent refinement of the PtDA will take place.

\section{Patient and public involvement}

A patient representative who had prior experience of the decision at question in our PtDA was recruited to the steering committee prior to protocol development. Our representative contributed to overall protocol development, particularly the feasibility of stage 3 to the public. It was anticipated our patient representative would contribute heavily to the design and format of the PtDA, commenting on readability, layout and presentation of information. Patient representative did not contribute to study recruitment. Patients will also be involved in questionnaire development (stage 1) and design via a focus group.

\section{ETHICS AND DISSEMINATION Ethical considerations}

Research ethics approval from North East-Tyne \& Wear South Research Ethics Committee (Ref: 19/NE/0073) and Health Research Authority approval (Ref: 257044) have been granted. Written, signed consent will be obtained from all participants at stages where it is necessary. Participants will have the right to withdraw from the research process at any time throughout the study. All interviews will be kept strictly confidential and patients and healthcare professionals will be given a study ID number to maintain this confidentiality. Participation in this study will not interfere with usual patient care.

\section{Dissemination}

This is a multidisciplinary, collaborative project with clinicians and patients. This will allow us to disseminate the research and its milestones into both the National Health Service (NHS) and the wider healthcare community through a variety of local, national and international forums such as charities and international meetings. We will also seek to index an online version of the PtDA in the Decision Aid Library Inventory once we have completed and analysed the paper version. We will also seek to index the NHS library of decision aids. A web-based version of the PtDA will also allow dissemination through the internet which is widely accessible to patients worldwide. Social media such as Twitter and blogs can also be used to signpost the availability of the instrument to both clinicians and patients.

We plan to impact the academic and clinical community more widely through a combination of conference presentations and peer-reviewed publications.

The primary output of this study is the PtDA, available in print. This will then be evaluated for effectiveness in a larger study, outside the costings of this grant application. Once fully evaluated it will be promoted more widely through social media, charity websites, professional organisations and academic sources.

\section{Author affiliations}

${ }^{1}$ Leicester Medical School, Leicester, UK

${ }^{2}$ Department of General Surgery, Sheffield Teaching Hospitals NHS Foundation Trust, Sheffield, UK

${ }^{3}$ Department of Psychology, Leeds Beckett University, Leeds, UK

${ }^{4}$ ACPGBI Patient Liaison Group, London, UK

${ }^{5}$ Inflammatory Bowel Disease Nurse Specialist, Sheffield Teaching Hospitals NHS

Foundation Trust, Sheffield, UK

${ }^{6}$ Stoma Care Specialist Nurse, Sheffield Teaching Hospitals NHS Foundation Trust, Sheffield, UK

${ }^{7}$ Department of Gastroenterology, Hull and East Yorkshire Hospitals NHS Trust, Hull, UK

${ }^{8}$ Deparment of Psychology, Leeds Beckett University Faculty of Health and Social

Sciences, Leeds, UK

${ }^{9}$ Gastroenterology Unit, Sheffield Teaching Hospitals NHS Foundation Trust, Sheffield, UK

Twitter Daniel Mark Baker @dmbaker13 and Matthew James Lee @ wannabehawkeye

Contributors AJL is the chief investigator and secured grant funding with assistance from DMB, MJL, GLJ and SRB in formulating the protocol and attending grant interviews. AJL, DMB, MJL, GLJ, SRB, SS and SB provided intellectual input into the protocol for the grant application. AJL, DMB, MJL, GLJ, SRB, SS, SB, AMF, $\mathrm{KR}$ and RW provided intellectual input and study design for the final protocol for the study.

Funding This work was funded by the Crohn's and Colitis UK 'Living with IBD' research award for 2018. This was awarded to the research group, with AJL as the principal investigator.

Competing interests AJL is a consultant, advisory board member or received lecture fees for MSD, Janssen, Pfizer, Takeda Pharma, AbbVie, Dr Falk, Shield Pharmaceuticals and Vifor Pharma. All other authors have no conflicts of interest to declare.

Patient consent for publication Not required.

Provenance and peer review Not commissioned; externally peer reviewed.

Open access This is an open access article distributed in accordance with the Creative Commons Attribution Non Commercial (CC BY-NC 4.0) license, which 
permits others to distribute, remix, adapt, build upon this work non-commercially, and license their derivative works on different terms, provided the original work is properly cited, appropriate credit is given, any changes made indicated, and the use is non-commercial. See: http://creativecommons.org/licenses/by-nc/4.0/.

\section{ORCID iDs}

Daniel Mark Baker http://orcid.org/0000-0002-3708-790X

Matthew James Lee http://orcid.org/0000-0001-9971-1635

\section{REFERENCES}

1 Ordás I, Eckmann L, Talamini M, et al. Ulcerative colitis. The Lancet 2012;380:1606-19.

2 Sutherland L, Macdonald JK. Oral 5-aminosalicylic acid for induction of remission in ulcerative colitis. Cochrane Database Syst Rev 2006:CD000543.

3 Rutgeerts P, Sandborn WJ, Feagan BG, et al. Infliximab for induction and maintenance therapy for ulcerative colitis. N Engl J Med 2005;353:2462-76.

4 Sandborn WJ, van Assche G, Reinisch W, et al. Adalimumab induces and maintains clinical remission in patients with moderate-to-sever ulcerative colitis. Gastroenterology 2012;142:257-65.

5 Sandborn WJ, Su C, Sands BE, et al. Tofacitinib as induction and maintenance therapy for ulcerative colitis. N Engl J Med 2017;376:1723-36.

6 Cosnes J, Gower-Rousseau C, Seksik P, et al. Epidemiology and natural history of inflammatory bowel diseases. Gastroenterology 2011;140:1785-94.

7 Bohl JL, Sobba K. Indications and options for surgery in ulcerative colitis. Surg Clin North Am 2015;95:1211-32.

8 Siegel CA. Shared decision making in inflammatory bowel disease: helping patients understand the tradeoffs between treatment options. Gut 2012;61:459-65.

9 Peyrin-Biroulet L, Germain A, Patel AS, et al. Systematic review: outcomes and post-operative complications following colectomy for ulcerative colitis. Aliment Pharmacol Ther 2016;44:807-16.

10 Allison M, Lindsay J, Gould D, et al. Surgery in young adults with inflammatory bowel disease: a narrative account. Int J Nurs Stud 2013:50:1566-75.

11 Styliński R, Alzubedi A, Rudzki S. Parastomal hernia - current knowledge and treatment. Wideochir Inne Tech Maloinwazyjne 2018;13:1-8.

12 Elwyn G, Frosch D, Thomson R, et al. Shared decision making: a model for clinical practice. J Gen Intern Med 2012;27:1361-7.

13 sitecorel. Consent: Supported Decision-Making - a good practice guide - Royal College of Surgeons [Internet]. Royal College of Surgeons. Available: https://www.rcseng.ac.uk/library-andpublications/rcs-publications/docs/consent-good-practice-guide/ [Accessed cited 7 Nov 2018].

14 Ulcerative colitis: management | Guidance and guidelines | NICE. Available: https://www.nice.org.uk/guidance/cg166/evidence [Accessed cited 7 Nov 2018]

15 Byrne CM, Tan K-K, Young JM, et al. Patient and clinician preferences for surgical and medical treatment options in ulcerative colitis. Colorectal Dis 2014;16:285-92.

16 Engelhardt EG, Pieterse AH, van der Hout A, et al. Use of implicit persuasion in decision making about adjuvant cancer treatment: a potential barrier to shared decision making. Eur J Cancer 2016;66:55-66.

17 Siegel CA. Embracing the Internet for progress in shared decisionmaking. Inflamm Bowel Dis 2007;13:1579-80.

18 Stacey D, Légaré F, Lewis K, et al. Decision AIDS for people facing health treatment or screening decisions. Cochrane Database Syst Rev 2017;4:CD001431.

19 Stacey D, Bennett CL, Barry MJ, et al. Decision AIDS for people facing health treatment or screening decisions. Cochrane Database Syst Rev 2011:CD001431.

20 Bekker HL, Winterbottom AE, Butow P, et al. Do personal stories make patient decision AIDS more effective? A critical review of theory and evidence. BMC Med Inform Decis Mak 2013;13:S9.

21 Knops AM, Legemate DA, Goossens A, et al. Decision AIDS for patients facing a surgical treatment decision: a systematic review and meta-analysis. Ann Surg 2013;257:860-6.

22 Patient Decision Aids - Ottawa Hospital Research Institute [Internet] Available: https://decisionaid.ohri.ca/AZlist.html [Accessed cited 7 Nov 2018].
23 Baker DM, Marshall JH, Lee MJ, et al. A systematic review of Internet decision-making resources for patients considering surgery for ulcerative colitis. Inflamm Bowel Dis 2017;23:1293-300.

24 Coulter A, Stilwell D, Kryworuchko J, et al. A systematic development process for patient decision AIDS. BMC Med Inform Decis Mak 2013;13:S2.

25 Jones GL, Hughes J, Mahmoodi N, et al. Observational study of the development and evaluation of a fertility preservation patient decision aid for teenage and adult women diagnosed with cancer: the cancer, fertility and me research protocol. BMJ Open 2017;7:e013219.

26 Baker DM, Lee MJ, Jones GL, et al. The informational needs and preferences of patients considering surgery for ulcerative colitis: results of a qualitative study. Inflamm Bowel Dis 2018;24:179-90.

27 Joseph-Williams N, Newcombe R, Politi M, et al. Toward minimum standards for certifying patient decision AIDS: a modified Delphi consensus process. Med Decis Making 2014;34:699-710.

28 Burton M, Kilner K, Wyld L, et al. Information needs and decisionmaking preferences of older women offered a choice between surgery and primary endocrine therapy for early breast cancer. Psychooncology 2017;26:2094-100.

29 Degner LF, Sloan JA, Venkatesh P. The control preferences scale. Can J Nurs Res 1997;29:21-43.

30 Abhyankar P, Volk RJ, Blumenthal-Barby J, et al. Balancing the presentation of information and options in patient decision AIDS: an updated review. BMC Med Inform Decis Mak 2013;13:S6.

31 Trevena LJ, Barratt A, Butow P, et al. A systematic review on communicating with patients about evidence. J Eval Clin Pract 2006:12:13-23.

32 Trevena LJ, Zikmund-Fisher BJ, Edwards A, et al. Presenting quantitative information about decision outcomes: a risk communication primer for patient decision aid developers. BMC Med Inform Decis Mak 2013;13:S7.

33 Barratt A, Trevena L, Davey HM, et al. Use of decision AIDS to support informed choices about screening. BMJ 2004;329:507-10.

34 Fagerlin A, Pignone M, Abhyankar P, et al. Clarifying values: an updated review. BMC Med Inform Decis Mak 2013;13:S8.

35 Ley P, Florio T. The use of readability formulas in health care. Psychol Health Med 1996;1:7-28.

36 How to Write Easy-to-Read Health Materials: MedlinePlus [Internet]. Available: https://medlineplus.gov/etr.html [Accessed cited $15 \mathrm{Nov}$ 2018].

37 Doak LG, Doak CC, Meade CD. Strategies to improve cancer education materials. Oncol Nurs Forum 1996;23:1305-12.

38 Baker SE, Edwards R, Doidge M. How many qualitative interviews is enough? Expert voices and early career reflections on sampling and cases in qualitative research. Southampton, UK: National Centre for Research Methods, 2012.

39 Guest G, Bunce A, Johnson L. How many interviews are enough?: an experiment with data saturation and variability. Field Methods 2006;18:59-82.

40 Braun V, Clarke V. Using thematic analysis in psychology. Qual Res Psychol 2006;3:77-101.

41 Bennett C, Graham ID, Kristjansson E, et al. Validation of a preparation for decision making scale. Patient Educ Couns 2010;78:130-3.

42 McGillion M, Carroll S, Metcalfe K, et al. Development of a patient decision aid for people with refractory angina: protocol for a threephase pilot study. Health Qual Life Outcomes 2014;12:93.

43 Elwyn G, Scholl I, Tietbohl C, et al. "Many miles to go ...": a systematic review of the implementation of patient decision support interventions into routine clinical practice. BMC Med Inform Decis Mak 2013;13:S14.

44 Marteau TM, Bekker H. The development of a six-item short-form of the state scale of the Spielberger State-Trait anxiety inventory (STAI). Br J Clin Psychol 1992;31:301-6.

45 Stage of Decision Making - Evaluation Measures - Patient Decision Aids - Ottawa Hospital Research Institute [Internet]. Available: https://decisionaid.ohri.ca/eval_stage.html [Accessed cited 7 Nov 2018].

46 O'Connor AM. Validation of a decisional conflict scale. Med Decis Making 1995;15:25-30.

47 Carrying Out Qualitative Analysis" IN: Ritchie, J. and Lewis, J. Qualitative research practice: a guide for social science students and researchers, pp. 219-262 | Manchester Metropolitan University [Internet]. Available: https://mmu.rl.talis.com/items/66AF60B5E551-9DB6-49A9-F7A51E02C076.html [Accessed cited 15 Nov 2018]. 